\title{
Microwave-induced spin-flip scattering of electrons in point contacts
}

\author{
A.M. Kadigrobov ${ }^{1,2}$, R.I. Shekhter ${ }^{1}$, I. Aronov ${ }^{3}$, S.I. Kulinich ${ }^{1,4}$, A. Pulkin ${ }^{1}$, and M. Jonson ${ }^{1,5,6}$ \\ ${ }^{1}$ Department of Physics, University of Gothenburg, SE-412 96 Göteborg, Sweden \\ E-mail: anatoli.kadigrobov@physics.gu.se \\ ${ }^{2}$ Theoretische Physik III, Ruhr-Universität Bochum, D-44801 Bochum, Germany \\ ${ }^{3} 2366$ Wilderness Way, Marietta, GA 30066, USA \\ ${ }^{4}$ B. Verkin Institute for Low Temperature Physics and Engineering of the National Academy of Sciences of Ukraine \\ 47 Lenin Ave., Kharkov 61103, Ukraine \\ ${ }^{5}$ SUPA, Department of Physics, Heriot-Watt University, Edinburgh EH14 4AS, Scotland, UK \\ ${ }^{6}$ Division of Quantum Phases and Devices, School of Physics, Konkuk University, Seoul 143-701, Korea
}

Received April 5, 2011

\begin{abstract}
We investigate resonant interaction of conduction electrons with an electromagnetic field that irradiates a point contact between a ferromagnetic and a normal metal in the presence of a strong magnetic field of order $1 \mathrm{~T}$. We show that electron spin-flips caused by resonant absorption and stimulated emission of photons result in a sharp peak in the magnetic-field dependence of the point-contact resistance. The height of the peak is shown to be directly proportional to the net rate of energy transfer to the electromagnetic field in the point contact due to absorption and stimulated emission of photons. Estimations indicate that our theory can serve as a basis for the explanation of recent experiments [A.M. Kadigrobov et al., New J. Phys. 13, 023007 (2011)].

PACS: 72.25.-b Spin polarized transport;

73.40.Jn Metal-to-metal contacts;

75.76. $+\mathbf{j}$ Spin transport effects;

85.75.-d Magnetoelectronics, spintronics, devices exploiting spin polarized transport or integrated magnetic field.
\end{abstract}

Keywords: point-contact spectroscopy, hot electrons, stimulated photon emission, spin-polarized injection.

\section{Introduction}

Forcing a current to go through a nanoconstriction is one of the most efficient methods available for creating highly nonequilibrium distributions of electrons in metals. Electrical current densities as high as $10^{9} \mathrm{~A} / \mathrm{cm}^{2}$, which are unattainable in bulk metals, are easily achieved in point contacts with a diameter of a few nanometers [1]. The absence of Joule heating (which crucially limits electrical current densities in bulk metals) is ensured in microcontacts by the smallness of their size relative to the characteristic energy-relaxation length, $\lambda_{\varepsilon}$, of the "hot" electrons. The same condition is responsible for another remarkable feature of point contacts, which is that the excess energy of the hot electrons can be directly controlled by the voltage applied to the system. This is because the voltage drop occurs almost entirely in the point contact, since the current density quickly becomes negligible as the distance to the contact increases [1]. The ease by which the energy of hot electrons can be controlled makes it possible to scan the channels for inelastic electron scattering. This is the basis for point-contact spectroscopy of elementary excitations in metals and semiconductors [2,3].

Experiments with point contacts between two different metals present a new unique possibility for injecting hot electrons from one metal to the other and allow for comparative studies of the spectra of different materials in a single experiment. An important feature of heterocontacts is the 
focussing property of the point-contact interface, which leads to selective passage of hot electrons through the contacts depending on the orientation of their momenta (due to effects of refraction and total internal reflection) [4].

The electron spin does not effect the scattering of hot electrons if the point-contact metals are nonmagnetic. If magnetic point contacts are used, however, the electron spin degree of freedom becomes an essential element of the point-contact spectroscopy. This is because the exchange interaction shifts the energy levels of electrons with different spins with respect to each other. The spin-split energy levels may result in a highly spin-polarized electron system in the sense that both the total electron density and the electron density of states at the Fermi level may be very different for the different spin projections. Another consequence of the spin-splitting is that a new spectroscopic effect - electron-photon scattering - becomes possible in magnetic structures. In nonmagnetic metals electron-photon scattering is not allowed by the energy- and momentum conservation laws, but in magnetic point contacts such scattering manifests itself as a resonant process accompanied by electron spin-flips. This raises the question of the effect of this scattering on the electrical conductance of point contacts. The objective of our paper is to develop a theory of the electron-photon scattering in point contacts and its effect on the conductance. We are mainly concerned with the properties of magnetic contacts between two different magnetic metals or between one magnetic and one nonmagnetic one [5].

A microcontact between two different magnetic metals results in a "focusing" of injected hot electrons in spin space - an effect known as spin-dependent electron tunneling [6-9]. Due to this effect an excess spin density is accumulated in the point-contact region in conjunction with a concentration of the current. Under certain conditions such an accumulation is accompanied by an inverse population of the spin-split electron energy levels [10-12]. If this is the case, we show that electron-photon scattering results in the generation of coherent photons, which leads to an enhancement of the electromagnetic field propagating through the point contact. This effect has been observed experimentally [13]; in Sec. 5 we discuss the possibility of building a terahertz laser based on this phenomenon. Recently, photon emission from ferromagnetic metal junctions was also observed [14].

Below, we will first outline the formalism to be used for calculating the electrical current through the model point contact. The relative weakness of the electron-photon interaction then allows us to proceed in two steps. In the first step we calculate the inverse electron spin population accumulated in the contact region to zeroth order in the electron-photon interaction strength, while in the second step we find the induced photocurrent in the presence of radiation as well as the resulting change in the point-contact resistance to first order in the electron-photon interaction.

\section{Formulation of the problem}

The system under consideration is a point contact between two metals (labelled 1 and 2 in Fig. 1) under electromagnetic irradiation. Metal 1 is a ferromagnetic metal while metal 2 is either a ferromagnetic metal or a normal metal under an external magnetic field $H$. As illustrated in Fig. 1 the magnetization in metal 2 (or the external magnetic field) is assumed to be antiparallel to the magnetization in metal 1 . We will carry out the calculations for this case, but in Sec. 5 we will comment on the changes that occur if these directions instead are parallel to each other. We furthermore consider the point contact to be in the diffusive transport regime, so that the elastic mean free path $l$ is much shorter than the characteristic size $d$ of the contact.

In order to calculate the electrical current through the point contact (PC) one needs to find the distribution functions $f_{\sigma \mathbf{p}}^{(1,2)}(\mathbf{r})$ of electrons in metals 1 and 2 to the left and right of the constriction, respectively (see Fig. 1), where $\mathbf{p}$ is the electron momentum and $\sigma= \pm 1$ is the electron spin. In each of the metals these functions satisfy Boltzmann equations

$$
\begin{aligned}
& \mathbf{v}_{\sigma}^{(s)} \frac{\partial f_{\sigma \mathbf{p}}^{(s)}}{\partial \mathbf{r}}-e \frac{\partial \Phi^{(s)}}{\partial \mathbf{r}} \frac{\partial f_{\sigma \mathbf{p}}^{(s)}}{\partial \mathbf{p}}+\frac{f_{\sigma \mathbf{p}}^{(s)}-\left\langle f_{\sigma \mathbf{p}}^{(s)}\right\rangle_{s}}{t_{\sigma}^{(s)}}= \\
& =\sigma w_{\mathrm{ph}}^{(s)}\left\{f_{\uparrow}^{(s)}, f_{\downarrow \mathbf{p}}^{(s)}\right\}, \quad s=1,2 .
\end{aligned}
$$

Here $\mathbf{v}_{\sigma}^{(s)}(\mathbf{p})$ is the electron velocity in each metal, which is given by a momentum derivative of the electron energy as $\mathbf{v}_{\sigma}^{(s)}=\partial E_{\sigma}^{(s)}(\mathbf{p}) / \partial \mathbf{p}$, where

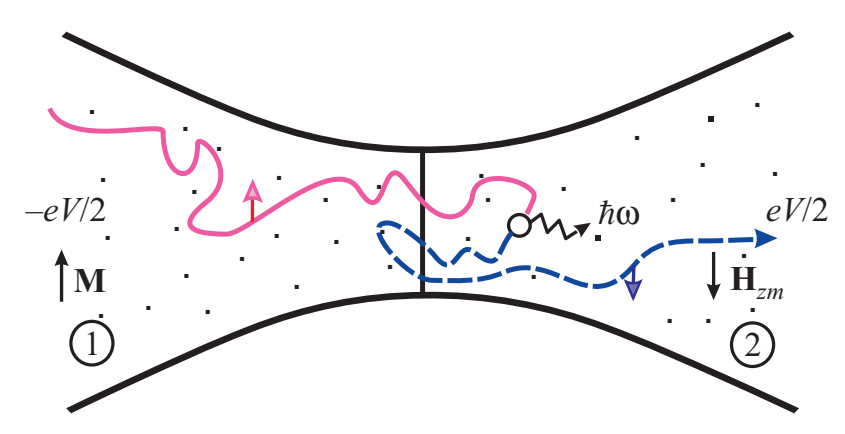

Fig. 1. Diffusive point contact under irradiation in the presence of a static magnetic field $\mathbf{H}$. A voltage bias $V$ injects a spin polarized current from a ferromagnetic metal (1) with magnetic moment $\mathbf{M}$ into a normal metal (2). A spin-up electron is shown to move along a diffusive trajectory from metal 1 to metal 2 (solid line) where it resonantly interacts with the electromagnetic field, which results in a spin flip and the emission of a photon. Continuing along its diffusive path with spin down (dash line) the spindependent contact resistance implies that the radiation induced spin-flip contributes to a change of the magnetoresistance of the point contact. 


$$
\begin{gathered}
E_{\sigma}^{(1)}(\mathbf{p})=\mathbf{p}^{2} / 2 m_{1}-\sigma J_{1}, \\
E_{\sigma}^{(2)}(\mathbf{p})=\mathbf{p}^{2} / 2 m_{2}+\sigma \mu_{B} H_{z m}
\end{gathered}
$$

are the electron dispersion laws and $m_{1}, m_{2}$ are the effective masses inside metals 1 and $2 ; J_{1}$ is the electron exchange energy in metal 1 while

$$
H_{z m}= \begin{cases}H, & \text { for normal metal } \\ J_{2} / \mu_{B}, & \text { for ferromagnetic metal } .\end{cases}
$$

Here $J_{2}$ is the electron exchange energy in metal 2 and $\mu_{B}$ is the Bohr magneton, $t_{\sigma}^{(s)}=l^{(s)} /\left|\mathbf{v}_{\sigma}^{(s)}\right|$ is the electron free path time and $l^{(s)}$ is the electron elastic relaxation length in metal $(s), \Phi^{(s)}$ is the electric potential; the notation $\langle\ldots\rangle_{s}$ implies an average of the bracketed quantity over the relevant Fermi surface,

$$
\langle\ldots\rangle_{s}=\int \frac{d S_{\mathbf{p}}}{\left|\mathbf{v}^{(s)}\right|}(\ldots) / \int \frac{d S_{\mathbf{p}}}{\left|\mathbf{v}^{(s)}\right|} .
$$

The electrical potential $\Phi^{(s)}(\mathbf{r})$ can be found from the electrical neutrality condition,

$$
\sum_{\sigma}\left\langle f_{\sigma \mathbf{p}}^{(s)}(\mathbf{r})-n_{F}\left(E_{\sigma}^{(s)}(\mathbf{p})\right)\right\rangle_{s}=0
$$

supplemented with the boundary conditions

$$
\Phi^{(s)}\left(z \rightarrow(-1)^{s} \infty\right)=(-1)^{s+1} V / 2,
$$

where $n_{F}$ is the Fermi function and $V$ is the applied voltage.

In the case that the electromagnetic field has a large amplitude the interaction of electrons with photons may be treated semi-classically, which allows the electron-photon collision integral to be written as

$$
\begin{aligned}
& w_{\mathrm{ph}}^{(s)}\left\{f_{\uparrow \mathbf{p}}^{(s)}, f_{\downarrow \mathbf{p}}^{(s)}\right\}=\frac{2 \pi}{\hbar} W^{2}\left[f_{\downarrow \mathbf{p}-\mathbf{q}}^{(s)}-f_{\uparrow \mathbf{p}}^{(s)}\right] \times \\
& \times \delta\left(E_{\uparrow}^{(s)}(\mathbf{p})-E_{\downarrow}^{(s)}(\mathbf{p}-\mathbf{q})-\hbar \omega\right) .
\end{aligned}
$$

Here $W$ is the matrix element of the coupling between the electron spin and an electromagnetic field of frequency $\omega$ and momentum $\mathbf{q}$,

$W= \begin{cases}\mu_{B} h_{a c}, & \text { for normal metal, } \\ \left(c / v_{F}\right)(J / \hbar \omega) \mu_{B} h_{a c}, & \text { for ferromagnetic metal } ;\end{cases}$

$J$ is the exchange interaction between conduction electrons and those of magnetic atoms in the magnetically ordered crystal, $v_{F}$ is the Fermi velocity, $h_{a c}$ is the amplitude of the magnetic component of the microwave field. Equation (3) reflects the fact that in nonmagnetic metals the electron spin and the electromagnetic field are coupled by the conventional relativistic Zeeman interaction, while in magnetic materials the exchange interaction of free electrons offers an efficient mechanism for radiation induced spin flips (see Ref. 10). This mechanism is based on the dependence of the exchange energy $J$, which is responsible for the Zeeman splitting, on the electronic momentum p [10]. The momentum dependence has to do with the overlap of the wave functions of the conduction electron and electrons of the magnetic subsystem (see, e.g., Ref. 15), which is determined by the value of $|\mathbf{p}| a / \hbar, a$ is the atomic spacing. In the presence of an electromagnetic field described by a vector potential $\mathbf{A}$ the momentum operator $\hat{\mathbf{p}}$ in the electron Hamiltonian must be changed to $\hat{\mathbf{p}}-(e / c) \mathbf{A}$. The resulting effective conduction electron spin-electromagnetic field interaction is presented in Eq. (8) (see Ref. 10 for details); the interaction strength, being proportional to the large exchange energy, exceeds the Zeeman interaction by orders of magnitude.

For the sake of simplicity we assume the scattering of electrons by the surface of the point contact $\Sigma_{P C}$ to be specular:

$$
f_{\sigma \mathbf{p}}^{(s)}\left(\mathbf{r} \in \boldsymbol{\Sigma}_{P C}\right)=f_{\sigma \mathbf{p}_{R}}^{(s)}\left(\mathbf{r} \in \boldsymbol{\Sigma}_{P C}\right),
$$

while the boundary conditions which couple the electron distribution functions at the interface $\boldsymbol{\Sigma}_{F F^{\prime}}$ between metals 1 and 2 can be written in the form

$$
\begin{aligned}
& f_{\sigma \mathbf{p}}^{(1)}=\left(1-D_{\sigma}\right) f_{\sigma \mathbf{p}_{R}}^{(1)}+D_{\sigma} f_{\sigma \overline{\mathbf{p}}}^{(2)}, \\
& f_{\sigma \mathbf{p}}^{(2)}=D_{\sigma} f_{\sigma \mathbf{p}_{R}}^{(1)}+\left(1-D_{\sigma}\right) f_{\sigma \overline{\mathbf{p}}}^{(2)} .
\end{aligned}
$$

Here $D_{\sigma}=D_{\sigma}(\mathbf{p}, \overline{\mathbf{p}})$ is the spin-dependent transparency of the interface; $\mathbf{p}=\left(p_{\|}, p_{\perp}\right)$ and $\mathbf{p}_{R}=\left(p_{\|},-p_{\perp}\right)$ are the momenta of the incident and reflected electrons, respectively, while $p_{\|}$and $p_{\perp}$ are the projections of the electron momentum parallel and perpendicular to the scattering surface at the point of the reflection $\mathbf{r} \in \boldsymbol{\Sigma}_{P C}, \boldsymbol{\Sigma}_{F F^{\prime}}$ (we consider the case when the radii of the interface and the point-contact surface are much larger than the electron de Broglie wave length); the momentum of the transmitted electron $\overline{\mathbf{p}}$ is determined by the energy conservation condition, $E_{\sigma}^{1,2}(\mathbf{p})=E_{\sigma}^{2,1}(\overline{\mathbf{p}})$.

As the current spreads and its density decreases with an increased distance from the $\mathrm{PC}$, the electron system tends to the equilibrium state (as $|\mathbf{r}| \gg d$ ). Hence the electron distribution functions $f_{\sigma \mathbf{p}}^{(1,2)}$ must satisfy the boundary conditions

$$
f_{\sigma \mathbf{p}}^{(s)}(\mathbf{r} \rightarrow \pm \infty)=n_{F}\left(E_{\sigma}^{(s)}(\mathbf{p})\right) .
$$

We consider the limit of a weak electron-photon scattering, $d / l_{\mathrm{ph}} \ll 1$, where $l_{\mathrm{ph}}$ is the electron-photon relaxation length. In this limit it is possible to solve Eq. (1) by a perturbation expansion of the distribution functions $f_{\sigma \mathbf{p}}^{(s)}$ and the potential $\Phi^{(s)}$ in powers of the small parameter $d / l_{\mathrm{ph}}$, 


$$
\begin{aligned}
& f_{\sigma \mathbf{p}}^{(s)}=f_{\sigma \mathbf{p}, 0}^{(s)}+f_{\sigma \mathbf{p}, 1}^{(s)}+\ldots \\
& \Phi^{(s)}=\Phi_{0}^{(s)}+\Phi_{1}^{(s)}+\ldots
\end{aligned}
$$

where functions $f_{\sigma \mathbf{p}, 0}^{(s)}$ and $f_{\sigma \mathbf{p}, 1}^{(s)}$ satisfy the following set of equations:

$$
\begin{gathered}
\mathbf{v}_{\sigma}^{(s)} \frac{\partial f_{\sigma \mathbf{p}, 0}^{(s)}}{\partial \mathbf{r}}-e \frac{\partial \Phi_{0}^{(s)}}{\partial \mathbf{r}} \frac{\partial f_{\sigma \mathbf{p}, 0}^{(s)}}{\partial \mathbf{p}}+\frac{f_{\sigma \mathbf{p}, 0}^{(s)}-\left\langle f_{\sigma \mathbf{p}, 0}^{(s)}\right\rangle_{s}}{t_{\sigma}^{(s)}}=0, \\
\mathbf{v}_{\sigma}^{(s)} \frac{\partial f_{\sigma \mathbf{p}, 1}^{(s)}}{\partial \mathbf{r}}-e \frac{\partial \Phi_{0}^{(s)}}{\partial \mathbf{r}} \frac{\partial f_{\sigma \mathbf{p}, 1}^{(s)}}{\partial \mathbf{p}}+\frac{f_{\sigma \mathbf{p}, 1}^{(s)}-\left\langle f_{\sigma \mathbf{p}, 1}^{(s)}\right\rangle_{s}}{t_{\sigma}^{(s)}}= \\
=\sigma w_{\mathrm{ph}, 0}^{(s)}+e \frac{\partial \Phi_{1}^{(s)}}{\partial \mathbf{r}} \frac{\partial f_{\sigma \mathbf{p}, 0}^{(s)}}{\partial \mathbf{p}} .
\end{gathered}
$$

Here $w_{\mathrm{ph}, 0}^{(s)}=w_{\mathrm{ph}}^{(s)}\left\{f_{\uparrow \mathbf{p}, 0}^{(s)}, f_{\downarrow_{\mathbf{p}, 0}}^{(s)}\right\}$ is the electron-photon collision integral, Eq. (7), evaluated to zeroth order in the expansion parameter $d / l_{\text {ph }} \ll 1$.

Equations which determine the electrical potentials $\Phi_{0}^{(s)}(\mathbf{r})$ and $\Phi_{1}^{(s)}(\mathbf{r})$ are easily found from Eq. (5). As we do not need to solve these equations in order to find the current flowing throw the PC (see below), we do not present them here.

According to Eq. (12) the total current flowing through the PC, $I=I_{\mathrm{el}}+I_{\mathrm{ph}}$, is a sum of the elastic current $I_{\mathrm{el}}$ and the photocurrent $I_{\mathrm{ph}}$, which are given by the relations

$$
I_{\mathrm{el}(\mathrm{ph})}=\frac{e}{(2 \pi \hbar)^{3}} \sum_{\sigma} \int d \mathbf{S} \int d^{3} \mathbf{p} \mathbf{v}_{\sigma \mathbf{p}}^{(s)} f_{\sigma \mathbf{p}, 0(1)}^{(s)}(\mathbf{r}) .
$$

Here the first integration is over the cross-section area of the point contact.

In order to solve kinetic equations (13) and (14) we use the procedure developed in Refs. 4, 16, 17 after a straightforward generalization to the spin-polarized case. To zeroth order in the expansion parameter the distribution functions $f_{\sigma \mathbf{p}, 0}^{(s)}$ can be expressed as

$$
\begin{aligned}
& f_{\sigma \mathbf{p}, 0}^{(s)}(\mathbf{r})=\alpha_{\sigma \mathbf{p}}^{(s)}(\mathbf{r}) n_{F}\left(E_{\sigma}^{(s)}(\mathbf{p})+e \Phi_{0}^{(s)}(\mathbf{r})-e V / 2\right)+ \\
& +\left(1-\alpha_{\sigma \mathbf{p}}^{(s)}(\mathbf{r})\right) n_{F}\left(E_{\sigma}^{(s)}(\mathbf{p})+e \Phi_{0}^{(s)}(\mathbf{r})+e V / 2\right) .
\end{aligned}
$$

Here $\alpha_{\sigma \mathbf{p}}^{(1)}(\mathbf{r})$ and $\alpha_{\sigma \mathbf{p}}^{(2)}(\mathbf{r})$ are the probabilities for an electron moving from $-\infty$ along a diffusive trajectory (while its energy and spin projection are conserved) to arrive at point $\mathbf{r}$ in, respectively, metal 1 or 2 with momentum $\mathbf{p}$. The distribution functions $f_{\sigma \mathbf{p}}^{(2)}$ are sketched in Fig. 2.

Inserting Eq. (16) into Eq. (13) one finds that the factors $\alpha_{\sigma \mathbf{p}}^{(s)}$ satisfy the equation:

$$
\mathbf{v}_{\sigma}^{(s)} \frac{\partial \alpha_{\sigma \mathbf{p}}^{(s)}}{\partial \mathbf{r}}+\frac{\alpha_{\sigma \mathbf{p}}^{(s)}-\left\langle\alpha_{\sigma \mathbf{p}}^{(s)}\right\rangle}{t_{\sigma}^{(s)}}=0
$$

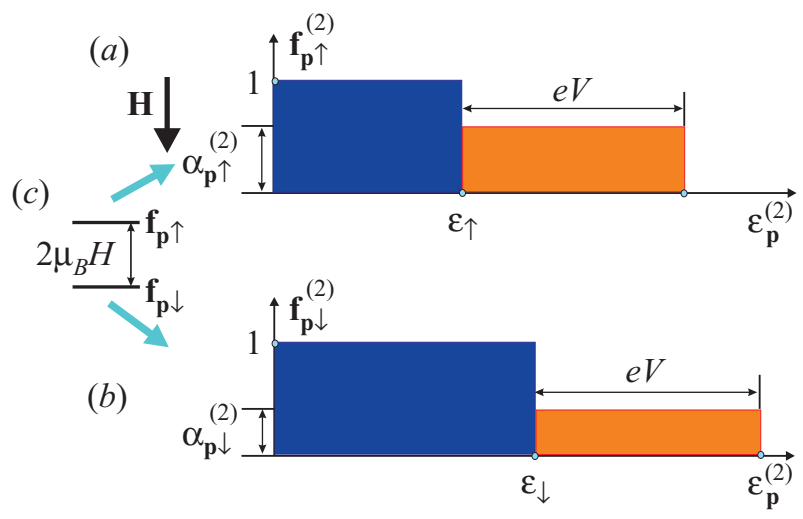

Fig. 2. Zero-temperature energy distributions for $(a)$ magnetic moment-up (spin-down), $\mathbf{f}_{\mathbf{p} \uparrow}$, and (b) magnetic moment-down (spinup) electrons, $\mathbf{f}_{\mathbf{p} \downarrow}$, at point $\mathbf{r}$ on the normal-metal side of the point contact. The inset $(c)$ shows the Zeeman energy splitting and the direction of the magnetic field $\mathbf{H}$. All states are occupied up to $\varepsilon \uparrow=\varepsilon_{f}-e V / 2-\mu_{B} H$ and $\varepsilon_{\downarrow}=\varepsilon_{f}-e V / 2+\mu_{B} H$, respectively (blue rectangles), but in the intervals $(\varepsilon \uparrow, \varepsilon \uparrow+e V)$ and $\left(\varepsilon_{\downarrow}, \varepsilon_{\downarrow}+e V\right)$ the states are only partly occupied (red rectangles) and to an extent that is determined by the probabilities $\alpha_{\uparrow}(\mathbf{p})$ and $\alpha_{\downarrow_{\mathbf{p}}}$ (r) for "hot" electrons in the ferromagnet to reach $\mathbf{r}$. Clearly, the difference between the densities of spin-down and spin-up electrons, $n_{\uparrow}(\mathbf{r})-n_{\downarrow}(\mathbf{r}) \propto\left[\left(\alpha_{\uparrow}^{(2)}-\alpha_{\downarrow}^{(2)}\right) e V-2 \mu_{B} H\right]$, depends on the bias voltage $V$. It follows that the spin population can be inverted, so that $n_{\uparrow}(\mathbf{r})>n_{\downarrow}(\mathbf{r})$, for large enough $V$ if $\alpha_{\uparrow}^{(2)}>\alpha_{\downarrow}^{(2)}$.

$\left\langle\alpha_{\sigma \mathbf{p}}^{(s)}\right\rangle \equiv\left\langle\alpha_{\sigma \mathbf{p}}^{(s)}\right\rangle_{s}$. Here and below we assume $e V \ll \varepsilon_{F}$, which allows the term proportional to the electric field in Eq. (13) to be neglected.

The boundary conditions for $\alpha_{\sigma \mathbf{p}}^{(s)}(\mathbf{r})$ are

$$
\begin{gathered}
\alpha_{\sigma \mathbf{p}}^{(1)}(z \rightarrow-\infty)=1, \quad \alpha_{\sigma \mathbf{p}}^{(2)}(z \rightarrow+\infty)=0 ; \\
\alpha_{\sigma \mathbf{p}}^{(s)}\left(\mathbf{r} \in \Sigma_{P C}\right)=\alpha_{\sigma \mathbf{p}_{R}}^{(s)}\left(\mathbf{r} \in \Sigma_{P C}\right),
\end{gathered}
$$

where the boundary condition at the interface between metals 1 and 2 follow from Eq. (10).

To first order in the expansion parameter $d / l^{(s)} \ll 1$, it follows from Eq. (17) that

$$
\alpha_{\sigma \mathbf{p}}^{(s)}=\left\langle\alpha_{\sigma \mathbf{p}}^{(s)}\right\rangle-l^{(s)} \mathbf{n}_{\sigma}^{(s)} \frac{\partial\left\langle\alpha_{\sigma \mathbf{p}}^{(s)}\right\rangle}{\partial \mathbf{r}},
$$

where $l^{(s)}=\left|\mathbf{v}_{F \sigma}^{(s)}\right| t_{\sigma}^{(s)}$ is the mean free path of the electrons and $\mathbf{n}_{\sigma}^{(s)}=\mathbf{v}_{F \sigma}^{(s)} /\left|\mathbf{v}_{F \sigma}^{(s)}\right|$ is a unit vector along the Fermi velocity $\mathbf{v}_{F \sigma}^{(s)}$ of an electron with the spin $\sigma$. Here the isotropic part of $\alpha_{\sigma \mathbf{p}}^{(s)}$ satisfies the diffusion equation

$$
v_{i} v_{k} \frac{\partial^{2}}{\partial \mathbf{r}_{i} \partial \mathbf{r}_{k}}\left\langle\alpha_{\sigma \mathbf{p}}^{(s)}\right\rangle=0
$$

with boundary conditions $\left\langle\alpha_{\sigma \mathbf{p}}^{(1)}(z \rightarrow-\infty)\right\rangle=1 \quad$ and $\left\langle\alpha_{\sigma \mathbf{p}}^{(2)}(z \rightarrow+\infty)\right\rangle=0$ far from the PC. In the vicinity of the 
F/N interface $\left(\mathbf{r} \in \boldsymbol{\Sigma}_{F N}\right)$ the effective boundary conditions are (see Ref. 4)

$$
\begin{gathered}
\left\langle\alpha_{\sigma \mathbf{p}}^{(2)}\right\rangle-\left\langle\alpha_{\sigma \mathbf{p}}^{(1)}\right\rangle=\frac{l^{(1)}}{Q_{\sigma}^{(1)}} \mathbf{e}_{\perp} \frac{\partial\left\langle\alpha_{\sigma \mathbf{p}}^{(1)}\right\rangle}{\partial \mathbf{r}}, \\
\frac{l^{(1)}}{Q_{\sigma}^{(1)}} \mathbf{e}_{\perp} \frac{\partial\left\langle\alpha_{\sigma \mathbf{p}}^{(1)}\right\rangle}{\partial \mathbf{r}}=\frac{l^{(2)}}{Q_{\sigma}^{(2)}} \mathbf{e}_{\perp} \frac{\partial\left\langle\alpha_{\sigma \mathbf{p}}^{(2)}\right\rangle}{\partial \mathbf{r}},
\end{gathered}
$$

where $\mathbf{e}_{\perp}$ is a unit vector perpendicular to the interface at point $\mathbf{r} \in \boldsymbol{\Sigma}_{F N}$, and

$$
Q_{\sigma}^{(s)}=\frac{\left\langle D_{\sigma}\right\rangle_{s^{\prime}}\left\langle n_{s^{\prime}} D_{\sigma}\right\rangle_{s}+\left\langle D_{\sigma}\right\rangle_{s}\left(1-\left\langle n_{s^{\prime}} D_{\sigma}\right\rangle_{s^{\prime}}\right)}{\left(1-\left\langle n_{s} D_{\sigma}\right\rangle_{s}\right)\left(1-\left\langle n_{s^{\prime}} D_{\sigma}\right\rangle_{s^{\prime}}\right)-\left\langle n_{s} D_{\sigma}\right\rangle_{s^{\prime}}\left\langle n_{s^{\prime}} D_{\sigma}\right\rangle_{s}} .
$$

Here $n_{s}=\left|\mathbf{e}_{\perp} \mathbf{n}_{\sigma}^{s}\right|$, and $s^{\prime} \neq s$. It is clear from Eq. (22) that for a low barrier transparency, $D_{\sigma} \ll 1$, one has

$$
Q_{\sigma}^{(s)} \approx\left\langle D_{\sigma}\right\rangle_{s}
$$

in the opposite limit of a high transparency barrier, $\delta D_{\sigma}=1-D_{\sigma} \ll 1$, one has

$$
Q_{\sigma}^{(s)} \approx \frac{1}{\left\langle n_{s} \delta D_{\sigma}\right\rangle_{s^{\prime}}+\left\langle n_{s^{\prime}} \delta D_{\sigma}\right\rangle_{s}} .
$$

For the sake of simplicity, we will below consider a point contact in the form of a channel, as shown in Fig. 3.

The channel is assumed to be long, $L \gg d \gg l$, where $L$ is the length of the channel and $d$ is its width. In this case the factor $\left\langle\alpha_{\sigma \mathbf{p}}^{(s)}(\mathbf{r})\right\rangle$ only depends on the coordinate $z$ along the channel and satisfies the equation

$$
\frac{d^{2}}{d z^{2}}\left\langle\alpha_{\sigma \mathbf{p}}^{(s)}(z)\right\rangle=0,
$$

with boundary conditions

$$
\left\langle\alpha_{\sigma \mathbf{p}}^{(1)}(z=-L / 2)\right\rangle=1,\left\langle\alpha_{\sigma \mathbf{p}}^{(2)}(z=L / 2)\right\rangle=0,
$$

together with the matching conditions in Eq. (21), where now $\mathbf{e}_{\perp} \partial / \partial \mathbf{r}=d / d z$

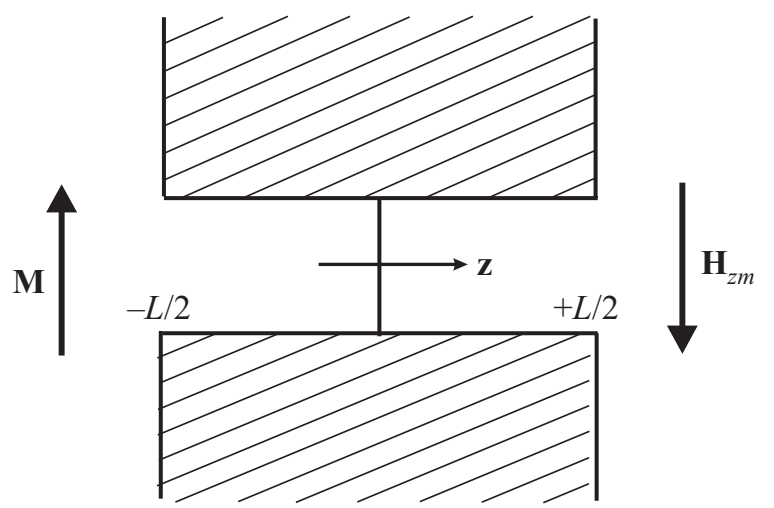

Fig. 3. Simplified point-contact geometry used for calculations (see text in Sec. 2).
The elementary solutions of the diffusion Eq. (25) with the above-mentioned boundary conditions are

$$
\begin{gathered}
\left\langle\alpha_{\sigma}^{(1)}(z)\right\rangle=1-\beta_{\sigma}^{(1)}\left(1+\frac{2 z}{L}\right), \\
\left\langle\alpha_{\sigma}^{(2)}(z)\right\rangle=\beta_{\sigma}^{(2)}\left(1-\frac{2 z}{L}\right) ;
\end{gathered}
$$

here we introduced the notations

$$
\beta_{\sigma}^{(s)}=\frac{\kappa_{\sigma}^{(s)}}{1+\kappa_{\sigma}^{(1)}+\kappa_{\sigma}^{(2)}} ; \quad \kappa_{\sigma}^{(s)}=Q_{\sigma}^{(s)} \frac{L}{2 l_{0}^{(s)}} .
$$

\section{Spin accumulation in the point contact}

If electrons are injected from metal 1 (i.e., if $\mathrm{eV}>0$ ) the numbers of "hot" electrons with spin up and down are

$$
\begin{gathered}
\delta n_{\sigma}=\int_{\Omega_{P C}^{(2)}} d^{3} \mathbf{r} \int \frac{d^{3} \mathbf{p}}{(2 \pi \hbar)^{3}} \times \\
\times\left[f_{\mathbf{p} \sigma, 0}^{(2)}(\mathbf{r})-n_{F}\left(E_{\sigma}^{(2)}(\mathbf{p})+e \Phi_{0}(\mathbf{r})+e V / 2\right)\right],
\end{gathered}
$$

where $\Omega_{P C}^{(2)}$ is the volume of the normal-metal part of the contact ("active" zone). Using Eq. (29) and Eqs. (16), and (19), (27) one finds

$$
\delta n_{\sigma}=\frac{\beta_{\sigma}^{(2)}}{4}\left(n_{0} \Omega_{P C}^{(2)}\right) \frac{e V}{\varepsilon_{F}},
$$

where $n_{0} \approx v_{F} \varepsilon_{F}$ is the conduction electron density in the normal metal $\left(v_{F}\right.$ is the electron density of states at the Fermi level $\varepsilon_{F}$ ). Therefore the total number of "hot" electrons in the normal-metal side of the contact is

$$
\delta n=\gamma_{\operatorname{tr}}\left(n_{0} \Omega_{P C}^{(2)}\right) \frac{e V}{\varepsilon_{F}}, \quad \gamma_{\operatorname{tr}}=\frac{\beta_{\uparrow}^{(2)}+\beta_{\downarrow}^{(2)}}{4} .
$$

As can be seen from Eq. (30) the induced magnetic moment corresponding to the net spin density accumulated in the same region is

$$
\delta M=\mu_{B} \beta_{\operatorname{tr}}\left(n_{0} \Omega_{P C}^{(2)}\right) \frac{e V}{\varepsilon_{F}}, \quad \beta_{\mathrm{tr}}=\frac{\beta_{\uparrow}^{(2)}-\beta_{\downarrow}^{(2)}}{4} .
$$

Notice, that the ratio $\beta_{\operatorname{tr}} / \gamma_{\operatorname{tr}}$ determines the effective spin of an injected electron,

$$
S=\frac{\delta n_{\uparrow}-\delta n_{\downarrow}}{\delta n}=\frac{\beta_{\uparrow}^{(2)}-\beta_{\downarrow}^{(2)}}{\beta_{\uparrow}^{(2)}+\beta_{\downarrow}^{(2)}},
$$

and, therefore, is a measure of the spin polarization of the "hot" electrons.

Thus the spin accumulation inside PC by the applied voltage $V$ produces an additional moment inside the PC. If $d$ is of the size of several hundred $\mathrm{nm}$ and $\beta_{\mathrm{tr}} \approx 0.3$, 
which corresponds to a nearly ballistic $\mathrm{PC}, l \sim d$, and the spin polarization of $30 \%$ at the $\mathrm{F} / \mathrm{N}$ interface, one has

$$
\delta M \sim 10^{6} \mu_{B} \frac{e V}{\varepsilon_{F}} ; \delta n \sim 10^{9} \frac{e V}{\varepsilon_{F}} .
$$

As one sees from Eq. (28), the dependence of $\beta_{\sigma}^{(2)}$ on the spin polarization is much weakened if $\kappa_{\sigma}^{(s)} \ll 1$, which prevents spin accumulation.

Using Eq. (16) and Eqs. (19), (27), (28) for the distribution function $f_{\sigma \mathbf{p}, 0}^{(s)}(\mathbf{r})$ it is easy to obtain an expression for the conductance, $G=R^{-1}$, in the elastic limit. One finds that

$$
G=\frac{\pi G_{0}}{6} \frac{n_{0} \Omega_{P C}}{\varepsilon_{F}} \frac{\hbar v_{F}}{L} \sum_{\sigma} \frac{Q_{\sigma}^{(2)}}{1+\kappa_{\sigma}^{(1)}+\kappa_{\sigma}^{(2)}},
$$

where $G_{0}=2 e^{2} / h$ is the conductance quantum and $\Omega_{P C}$ is a point-contact volume.

\section{Photocurrent}

\subsection{Photocurrent through a point contact with one interface}

As the interaction of an electron with a photon does not essentially change the electron momentum (in contrast to the inelastic scattering of electrons by phonons and magnons) the main contribution to the photocurrent flowing through the point contact under electromagnetic irradiation arises due to the changes in the electron spin densities produced by vertical spin-flip transitions (in energymomentum space) stimulated by the photon field. The change of the electron distribution function $f_{\sigma \mathbf{p}, 1}^{(s)}(\mathbf{r})$ caused by these processes is obtained from the solution of Eq. (14) with the boundary conditions

$$
f_{\sigma \mathbf{p}, 1}^{(s)}\left(z \rightarrow(-1)^{s} \infty\right)=0, \Phi_{1}^{(s)}\left(z \rightarrow(-1)^{s} \infty\right)=0 .
$$

The matching conditions at the $\mathrm{F} / \mathrm{N}$ interface are given by Eq. (10) with the change $f_{\mathbf{\sigma p}}^{(s)} \rightarrow f_{\mathbf{\sigma p}, 1}^{(s)}$.

In the limit $\mathrm{eV} \ll \varepsilon_{F}$ one can neglect the terms proportional to the electric field on both sides of Eq. (14); the solution of this equation with the above-mentioned boundary conditions may be found using the Green's function,

$$
g_{\mathbf{p}, \mathbf{p}^{\prime}}^{(s, \sigma)}\left(\mathbf{r}, \mathbf{r}^{\prime}\right)=g_{-\mathbf{p}^{\prime},-\mathbf{p}}^{(s, \sigma)}\left(\mathbf{r}^{\prime}, \mathbf{r}\right) .
$$

This function satisfies the equations [17]

$$
\begin{gathered}
\mathbf{v}_{\sigma \mathbf{p}}^{(s)} \frac{\partial g_{\mathbf{p}, \mathbf{p}^{\prime}}^{(s)}\left(\mathbf{r}, \mathbf{r}^{\prime}\right)}{\partial \mathbf{r}} \\
+\frac{g_{\mathbf{p}, \mathbf{p}^{\prime}}^{(s, \sigma)}\left(\mathbf{r}, \mathbf{r}^{\prime}\right)-\left\langle g_{\mathbf{p}, \mathbf{p}^{\prime}}^{(s)}\left(\mathbf{r}, \mathbf{r}^{\prime}\right)\right\rangle_{s, \mathbf{p}^{\prime}}}{t_{\sigma}^{(s)}}= \\
=\delta\left(\mathbf{p}-\mathbf{p}^{\prime}\right) \delta\left(\mathbf{r}-\mathbf{r}^{\prime}\right),
\end{gathered}
$$

where $\mathbf{r}, \mathbf{r}^{\prime}$ are inside metal $s$ and $\langle\ldots\rangle_{s, \mathbf{p}^{\prime}}$ means averaging with respect to the direction of the momentum $\mathbf{p}^{\prime}$ (see
Eq. (4)). This equation is supplied with boundary conditions,

$$
\begin{gathered}
g_{\mathbf{p}, \mathbf{p}^{\prime}}^{(s, \sigma)}\left(z, z^{\prime} \rightarrow(-1)^{s} \infty\right)=0, \\
g_{\mathbf{p}, \mathbf{p}^{\prime}}^{(s, \sigma)}\left(\mathbf{r}, \mathbf{r}^{\prime} \in \boldsymbol{\Sigma}_{P C}\right)=g_{\mathbf{p}_{R}, \mathbf{p}^{\prime}}^{(s, \sigma)}\left(\mathbf{r}, \mathbf{r}^{\prime} \in \boldsymbol{\Sigma}_{P C}\right) ;
\end{gathered}
$$

at the $\mathrm{F} / \mathrm{N}$ interface $g_{\mathbf{p}, \mathbf{p}^{\prime}}^{(1, \sigma)}$ and $g_{\mathbf{p}, \mathbf{p}^{\prime}}^{(2, \sigma)}$ are matched with Eq. (10).

Using Eqs. (14), (38) and (15) one finds $f_{\sigma \mathbf{p}, 1}^{(s)}(\mathbf{r})$ and the photocurrent $I_{\mathrm{ph}}$,

$$
\begin{gathered}
f_{\sigma \mathbf{p}, 1}^{(s)}(\mathbf{r})=\sigma \int d \mathbf{r}^{\prime} \int d^{3} \mathbf{p}^{\prime} g_{\mathbf{p}, \mathbf{p}^{\prime}}^{(s, \sigma)}\left(\mathbf{r}, \mathbf{r}^{\prime}\right) w_{\mathrm{ph}, 0}^{(s)}\left(\mathbf{p}^{\prime}, \mathbf{r}^{\prime}\right), \\
I_{\mathrm{ph}}=e \sum_{s, \sigma} \sigma \int d \mathbf{r} \int \frac{d \mathbf{p}}{(2 \pi \hbar)^{3}} G_{\sigma \mathbf{p}}^{(s)}\left(\mathbf{r}, z^{\prime}\right) w_{\mathrm{ph}, 0}^{(s)}(\mathbf{p}, \mathbf{r}),
\end{gathered}
$$

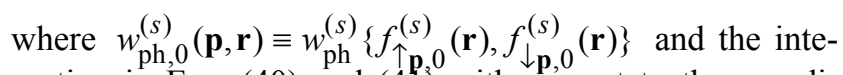
gration in Eqs. (40) and (41) with respect to the coordinates is over the volume $\Omega_{P S}^{(s)}$ of metal $s$; in Eq. (41) we introduced a new function $G_{\sigma \mathbf{p}}^{(s)}\left(\mathbf{r}, z^{\prime}\right)$, which has the form

$$
G_{\sigma \mathbf{p}}^{(s)}\left(\mathbf{r}, z^{\prime}\right)=\int d S^{\prime} \int d \mathbf{p}^{\prime} v_{z}^{(s)}\left(\mathbf{p}^{\prime}\right) g_{\mathbf{p}^{\prime}, \mathbf{p}}^{(s, \sigma)}\left(\mathbf{r}^{\prime}, \mathbf{r}\right),
$$

and the first integration is taken over the area of the pointcontact cross section taken at an arbitrary point $z=z^{\prime}$ inside metal $s$.

As follows from Eqs. (38), (39) and the symmetry property of Green's function, Eq. (37), the function $G_{\sigma p}^{(s)}\left(\mathbf{r}, z^{\prime}\right)$ is a solution of the equation,

$$
-\mathbf{v}_{\sigma \mathbf{p}}^{(s)} \frac{\partial G_{\sigma \mathbf{p}}^{(s)}}{\partial \mathbf{r}}+\frac{G_{\sigma \mathbf{p}}^{(s)}-\left\langle G_{\sigma \mathbf{p}}^{(s)}\right\rangle_{s}}{t_{\sigma}^{(s)}}=v_{z}^{(s)} \delta\left(z-z^{\prime}\right),
$$

subject to the boundary conditions

$$
G_{\sigma \mathbf{p}}^{(s)}\left(z \rightarrow(-1)^{s} \infty, z^{\prime}\right)=0 .
$$

Comparing Eq. (43) and Eq. (17) and their boundary conditions one finds that

$$
G_{\sigma \mathbf{p}}^{(s)}(\mathbf{r}, z)=\alpha_{\sigma,-\mathbf{p}}^{(s)}+\theta\left(z^{\prime}-z\right) .
$$

The last term on the right-hand side of Eq. (45) does not contribute to Eq. (41), and therefore the photocurrent can be rewritten in terms of the probabilities $\alpha_{\sigma, \mathbf{p}}(\mathbf{r})$ for an arbitrary form of the point contact as

$$
\begin{gathered}
I_{\mathrm{ph}}=e \sum_{s} \int_{\Omega_{P C}^{(s)}} d \mathbf{r} \int \frac{d \mathbf{p}}{(2 \pi \hbar)^{3}} \times \\
\times\left(\alpha_{\uparrow,-\mathbf{p}}^{(s)}(\mathbf{r})-\alpha_{\downarrow,-\mathbf{p}}^{(s)}(\mathbf{r})\right) w_{\mathrm{ph}}^{(s)}\left\{f_{\uparrow \mathbf{p}, 0}^{(s)}, f_{\downarrow \mathbf{p}, 0}^{(s)}\right\} .
\end{gathered}
$$

Using Eqs. (46), (27) and (28) one obtains the total current $I(V)$ in a diffusive point contact under irradiation as 


$$
\begin{gathered}
I(V)=\frac{V}{R}+\theta\left(\frac{v_{F}}{c}-\frac{\left|\hbar \omega-2 \mu_{B} z\right|}{\hbar \omega}\right) j_{\mathrm{ph}}(V), \\
j_{\mathrm{ph}}(V)=\frac{\Delta R}{R^{2}}\left(V-V_{*}\right), e V_{*}=\frac{3}{8} \frac{\hbar \omega}{\beta_{\mathrm{tr}}} .
\end{gathered}
$$

Here $R$ is the "dark" contact resistance, Eq. (35), while the relative change of the point-contact resistance caused by the irradiation is

$$
\frac{\Delta R}{R}=\frac{\left(4 \pi \beta_{\mathrm{tr}}\right)^{2}}{6} \frac{c}{v_{F}} \frac{W^{2}}{\varepsilon_{F} \hbar \omega}\left(n_{0} \Omega_{P C}^{(2)}\right)\left(\frac{2 e^{2}}{h} R\right) .
$$

As one sees from Eq. (47) the dependence of the photocurrent on the magnetic field has a sharp peak corresponding to the resonant interaction of electron spins and the electromagnetic field.

\subsection{Photocurrent through a point contact with two interfaces}

The possibility to detect electromagnetic radiation by measuring the current that flows through the point contact under consideration in this work is based on the dependence of the resistance of the interface between the normal and ferromagnetic metals (or between two ferromagnetic metals) on the spin orientation of transmitted electrons. The physical mechanism will be outlined in more detail below.

An electron injected from the ferromagnetic metal into the normal metal moves along a diffusive trajectory while being scattered by impurities. The result is an electronic back-flow, since some injected electron returns to the injecting interface. Thus, a repeated crossing of the interface can be expected due to the presence of impurities. If no photon emission takes place the electron returns to the injecting interface with the same spin orientation. On the other hand, if a spin-flip photon emission occurs, then the electron back-flows with the opposite spin orientation and therefore encounters a different interface resistance. This change in interface resistance for the back-flow current can be detected by measuring the changes in the current-voltage characteristics upon irradiation. From this consideration it is clear that the presence of impurities is important for detecting the effect using transport spectroscopy (this conclusion is confirmed by Eq. (46) for the photocurrent as the latter goes to zero along with $\beta_{\sigma}^{(2)}$ as $l_{2} \rightarrow \infty$ (see Eq. (28)). On the other hand, the photoemission effect is enhanced along with the increase of the pumping current through ballistic contacts, at the same time becoming more difficult for detection with the help of transport measurements in the above geometry.

In this subsection we introduce a modification of the set-up that allows the electron-photon relaxation to be detected also in ballistic point contacts (see Fig. 4), where the effect should be more prominent. The detection method in

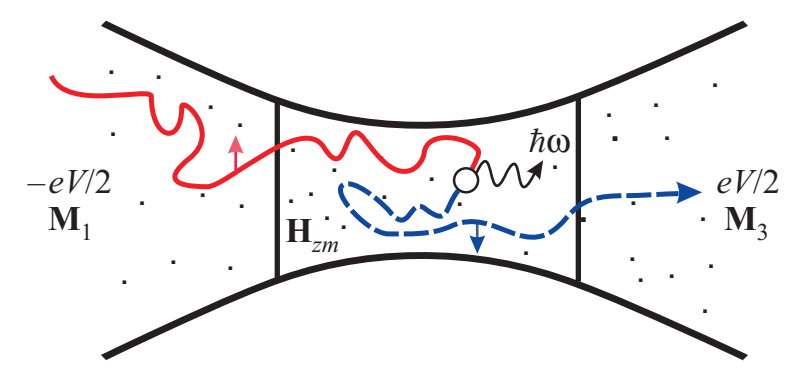

Fig. 4. Diffusive point contact under irradiation in the presence of a static magnetic field $H$. A voltage bias $V$ injects a spin polarized current from a ferromagnetic metal with magnetic moment $M_{1}$ into a normal metal. A spin-up electron is shown to move along a diffusive trajectory from metal 1 to metal 2 (solid line) where it resonantly interacts with the electromagnetic field. This interaction results in a spin flip and the emission of a photon. Continuing along its diffusive path with spin down (dash line) the electron crosses the second interface and the spin-dependent contact resistance implies that the radiation induced spin-flip contributes to a change of the magnetoresistance of the point contact.

this $\mathrm{F} / \mathrm{N} / \mathrm{F}$ (or $\mathrm{F} / \mathrm{F}_{1} / \mathrm{F}$ ) geometry relies on the forward-flow current rather than the back-flow current as is the case for the single-interface PC considered earlier.

Figure 4 illustrates the two-interfaces magnetic pointcontact geometry. Here the electron that undergoes electron-photon scattering preserves its momentum and traverses the second interface, the resistance of which is spin dependent and therefore sensitive to whether a spin-flip even has occurred. The point contact under consideration consists of three conductors with two ferromagnetic electrodes aligned either parallel or antiparallel.

The current through the point contact to be

$$
\begin{gathered}
I(V)=\frac{V}{R}+\theta\left(\frac{v_{F}}{c}-\frac{\left|\hbar \omega-2 \mu_{B} H_{z m}\right|}{\hbar \omega}\right) j_{\mathrm{ph}}(V), \\
j_{\mathrm{ph}}(V)=\frac{\Delta R}{R^{2}}\left(V-V_{*}\right), e V_{*}=\frac{\alpha_{-}}{\alpha_{-}^{2}+b_{\mathrm{tr}}^{2} / 3} \hbar \omega .
\end{gathered}
$$

Here the change of the point-contact resistance is

$$
\frac{\Delta R}{R}=\pi^{2}\left(\alpha_{-}^{2}+b_{\mathrm{tr}}^{2} / 3\right) \frac{c}{v_{F}} \frac{W^{2}}{\varepsilon_{F} \hbar \omega}\left(n_{0} \Omega_{P C}^{(2)}\right)\left(\frac{2 e^{2}}{h} R\right),
$$

where $\Omega_{P C}^{(2)}$ is the volume of the part of the point contact between the barriers; $W$ is given by Eq. (8), and

$$
\begin{gathered}
\alpha_{-}=\frac{1}{2} \sum_{\sigma= \pm 1} \sigma \frac{\eta_{\sigma}^{R, 2}-\eta_{\sigma}^{L, 2}+K_{\sigma}^{(-)}}{\eta_{\sigma}^{R, 2}+\eta_{\sigma}^{L, 2}+K_{\sigma}^{(+)}+2}, \\
b_{\mathrm{tr}}=\sum_{\sigma= \pm 1} \frac{\sigma}{\eta_{\sigma}^{R, 2}+\eta_{\sigma}^{L, 2}+K_{\sigma}^{(+)}+2},
\end{gathered}
$$


while

$$
K_{\sigma}^{( \pm)}=\frac{\eta_{\sigma}^{R, 2}}{\eta_{\sigma}^{R, 3}} \pm \frac{\eta_{\sigma}^{L, 2}}{\eta_{\sigma}^{L, 1}}
$$

Here

$$
\begin{gathered}
\eta_{\sigma}^{L, 2}=l_{2} / L_{2} Q_{\sigma}^{(L, 2)}, \eta_{\sigma}^{R, 2}=l_{2} / L_{2} Q_{\sigma}^{(R, 2)}, \\
\eta_{\sigma}^{L, 1}=l_{1} / L_{1} Q_{\sigma}^{(L, 1)}, \eta_{\sigma}^{R, 3}=l_{3} / L_{3} Q_{\sigma}^{(R, 3)},
\end{gathered}
$$

where $l_{i}(i=1,2,3)$ are the electron free path lengths and $L_{i}(i=1,2,3)$ are the lengths of the parts of the point contact separated by the left and right interfaces which have transparencies $D_{\mathbf{p}}^{(L)}$ and $D_{\mathbf{p}}^{(R)}$ (see Fig. 5); $Q^{(L, R ; s)}$ are defined by Eq. (22) in which $D_{\mathbf{p}}$ should be changed to $D_{\mathbf{p}}^{(L, R)}$ in such a way that $Q_{\sigma}^{(s)} \rightarrow Q_{\sigma}^{(L, s)}$ with $s \neq s^{\prime}=1,2$ and $Q_{\sigma}^{(s)} \rightarrow Q_{\sigma}^{(R, s)}$ with $s \neq s^{\prime}=2,3$.

If part 2 of the point contact between the barriers is "clean" while the external parts are "dirty", that is $l_{2} \gg\left(L_{2} / L_{1,3}\right) l_{1,3}$, and the transparencies of the barriers $D^{(L, R)} \sim 1$ one find $b_{\text {tr }} \ll 1$ and

$$
\alpha_{-} \approx \sum_{\sigma= \pm 1} \frac{\sigma}{1+\left(Q_{\sigma}^{(R, 2)} / Q_{\sigma}^{(L, 2)}\right)\left(Q_{\sigma}^{(L, 1)} / Q_{\sigma}^{(R, 3)}\right)} .
$$

If the transparencies of the left and right interfaces are small, $D_{\sigma}^{L, R} \ll 1$ (here $D_{\sigma}^{L, R} \equiv\left\langle D_{\sigma, \mathbf{p}}^{L, R}\right\rangle_{s=2}$ ) and $\eta_{\sigma}^{L, R} \gg 1$ one has

$$
\alpha_{-} \approx \frac{1}{2} \sum_{\sigma= \pm 1} \sigma \frac{D_{\sigma}^{L}-D_{\sigma}^{R}}{D_{\sigma}^{L}+D_{\sigma}^{R}} ; b_{\mathrm{tr}} \approx 0 .
$$

Therefore, in the both cases the value of the photocurrent Eq. (49) does not depend on the electron mean free path inside the point contact and hence, in contrast to a point contact with one interface, it does not vanish in the ballistic limit in which the emission of photons and its effect on the point-contact electric transport properties are maximal.

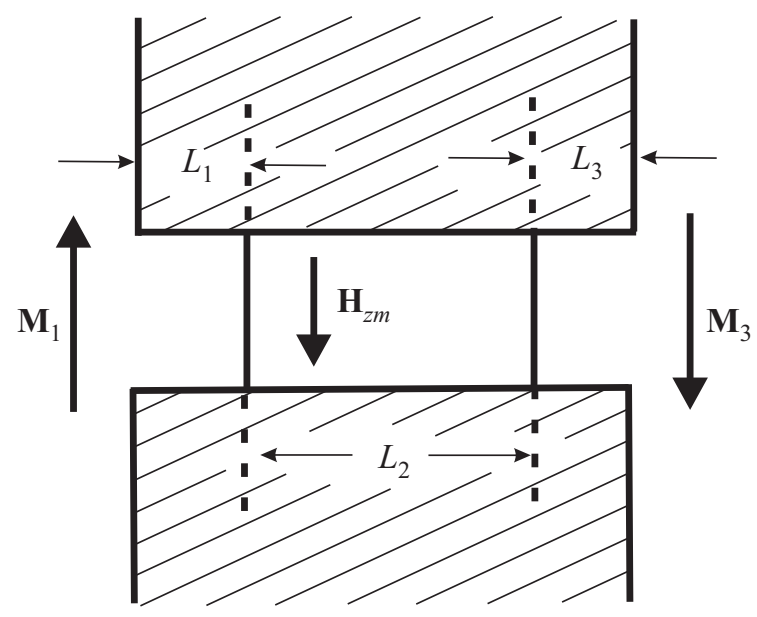

Fig. 5. Simplified geometry of the point contact with two interfaces used for calculations in Sec. 4.2.

\subsection{Net emitted power in the irradiated point contact}

A comparison between Eq. (46) and the rate equation for photons generated by electronic spin-flip transitions induced by the electromagnetic field (see Ref. 10),

$$
\frac{d n_{\mathrm{ph}}^{(s)}}{d t}=-\int w_{\mathrm{ph}}^{(s)}\left\{f_{\uparrow \mathbf{p}}^{(s)}, f_{\downarrow \mathbf{p}}^{(s)}\right\} \frac{d^{3} \mathbf{p}}{(2 \pi \hbar)^{3}},
$$

where $n_{\mathrm{ph}}$ is the photon density, shows that the photocurrent may be rewritten in the form

$$
I_{\mathrm{ph}}=-e \sum_{s} \int_{\Omega^{(s)}} d \mathbf{r}\left(\left\langle\alpha_{\uparrow \mathbf{p}}^{(s)}\right\rangle-\left\langle\alpha_{\downarrow_{\mathbf{p}}}^{(s)}\right\rangle\right) \frac{d n_{\mathrm{ph}}^{(s)}}{d t},
$$

which makes it clear that its magnitude depends on the net rate of photon absorption/emission in combination with the spin dependence of the effective transparency of the point contact. From Eq. (47) one notes that the microwaveinduced current changes sign at $V=V_{*}$, i.e., when the rate of photon emission by "hot" electrons begins to exceed the rate of photon absorption.

The close relation between the electron transport and photon radiation processes allows us to express the photocurrent in terms of the power of emission and absorption of photons by electrons in the point contact. Using Eqs. (7), (16), and (27) one finds that the net emitted power due to resonant $\left(\hbar \omega=2 \mu_{B} H\right)$ absorption and emission of photons in the irradiated point contact, defined as $P(V)=\hbar \omega \int d \mathbf{r} d n_{\mathrm{ph}} / d t$, can be expressed as

$$
P(V)=P_{0}\left(-1+\frac{3}{4} \frac{V}{V_{*}}\right) .
$$

Here

$$
P_{0}=\frac{\pi \omega}{2 \varepsilon_{F}} \frac{c}{v_{F}}\left(n_{0} \Omega_{P C}^{(2)}\right) W^{2}
$$

is the absorbed power due to photon absorption at $V=0$. As is follows from Eq. (58) $V_{\text {th }}=(4 / 3) V_{*}$ is the threshold voltage at which the stimulated emission of photons from the point contact takes place.

Comparing Eq. (47) and Eq. (59) one finds that

$$
j_{\mathrm{ph}}(V)=\frac{3}{4} \frac{V-V_{*}}{V_{*}^{2}} P_{0},
$$

which makes it possible find the power $P_{0}$ absorbed from the electromagnetic field by measuring $d j_{\mathrm{ph}}(V) / d V$ (see Eq. (47)) after first having determined $V_{*}$ from the condition $j_{\mathrm{ph}}\left(V_{*}\right)=0$. Furthermore, the net emitted power $P(V)$ can be determined by measuring $j_{\mathrm{ph}}(V)$ with the help of Eq. (60) and Eq. (58).

\section{Conclusions}

We have shown that an inverted spin population is accumulated in a voltage biased point contact between a fer- 
romagnetic $(\mathrm{F})$ and a normal metal $(\mathrm{N})$. For a contact of linear dimension $d \sim 10 \mathrm{~nm}$, biased by a voltage $V$, and with a spin polarization of $30 \%$ at the $\mathrm{F} / \mathrm{N}$ interface $\left(\beta_{\mathrm{tr}} \sim 0.3\right)$ we find that the corresponding magnetic moment injected into the contact region is $\delta M \sim 10^{6} \mu_{B} e V / \varepsilon_{F}$. We have furthermore shown that if the point contact is irradiated by an electromagnetic field, photon-induced electron spin-flip scattering gives rise to a narrow peak in the radiation-frequency dependence of the relative change of the point-contact resistance, $\Delta R / R$. The peak appears when the frequency is resonant with the exchange splitting in the normal-metal spectrum of conduction electrons, which for an external magnetic field of $1 \mathrm{~T}$ occurs at $30 \mathrm{GHz}$.

The following features of the considered phenomenon should be mentioned. Being dependent on the total number of hot electrons with a given spin, the effect of electronphoton scattering does not depend on their energy distribution and hence the effect predicted here is not sensitive to the temperature. Our analysis shows that there is a special correlation between the relative orientation of the vectors $\mathbf{M}$ and $\mathbf{H}$ and the direction of the current injection needed for the observation of this phenomenon. Hence, in the case of an antiparallel orientation of $\mathbf{M}$ and $\mathbf{H}$ an injection of electrons into the nonmagnetic metal is needed to achieve a level of photon emission that is larger than the level of photon absorption, while in the case that $\mathbf{M}$ and $\mathbf{H}$ are parallel one needs the injection of electrons to be in the opposite direction, which can simply be achieved by reversing the voltage polarity.

The net power, $P(V)$, generated by the stimulated emission of photons in the electron spin-flip relaxation process can be determined by measuring the photon current $j_{\text {ph }}(V)$ defined in Eq. (47). In a typical experiment [18] the $12-48 \mathrm{~mW}$ of $14-64 \mathrm{GHz}$ radiation generated by an HF Klystron produces a magnetic amplitude $\left(h_{a c}\right)$ of the electromagnetic field inside the point contact of order $30 \mathrm{mT}$. For such a field we find that $\Delta R / R \sim 0.01-0.10 \%$ and that $P(V)$ is given by Eq. (58) with $P_{0} \sim 1-10 \mathrm{pW}$; $P_{0}$ being the power absorbed from the electromagnetic field due to photon absorption in the contact region. These estimates show that an experimental implementation of electromagnetic radiation spectroscopy in point contacts is quite feasible. Comparison between the above theoretical analysis and the recent observation by the ILT group shows a good quantitative agreement [13].

It follows from Eq. (58) that the radiation field is enhanced by the stimulated emission of photons if the proportionality coefficient is greater than zero, that is at $V>(4 / 3) V_{*}$. From here it follows that the point contact may be considered as an active element with the increment of the electromagnetic field given by

$$
\gamma=3 \pi^{2} \frac{c}{v_{F}} \frac{n_{0}}{\varepsilon_{F}} \mu_{B}^{2}\left(\frac{V}{V_{*}}-\frac{4}{3}\right) .
$$

Inserting typical parameters for the metal electrodes, one finds $\gamma \sim 2 \cdot 10^{-2}\left(V / V_{*}-4 / 3\right)$, and $V / V_{*}$ can reach as high as $\approx 10^{3}-10^{4}$ in realistic structures. This exceeds the increment that can be achieved in conventional semiconductor lasers by orders of magnitude and hence an array of such magnetic point contacts can be used as the active area of powerful lasers [19].

\section{Acknowledgments}

Financial support from the Swedish VR, the European Commission (FP7-ICT-2007-C; project No. 225955 STELE) and the Korean WCU program funded by MEST/NFR (R31-2008-000-10057-0) is gratefully acknowledged. SIK acknowledges the hospitality of the Department of Physics at the University of Gothenburg.

1. Y.G. Naidyuk and I.K. Yanson, Point Contact Spectroscopy, Springer Series in Solid-State Sciences 145, Springer, New York (2005).

2. A.G. Batrak and I.K. Yanson, Fiz. Nizk. Temp. 5, 1404 (1979) [Sov. J. Low Temp. Phys. 5, 663 (1979)].

3. I.O. Kulik, R.I. Shekhter, and A.N. Omel'yanchuk, Solid State Commun. 23, 301 (1977).

4. R.I. Shekhter and I.O. Kulik, Fiz. Nizk. Temp. 9, 46 (1983) [Sov. J. Low Temp. Phys. 9, 22 (1983)].

5. Electromagnetic radiation from a planar contact between a ferromagnetic and a nonmagnetic semiconductor in an external magnetic field was detected by N.A. Viglin, V.V. Osipov, A.A. Samokhvalov, and O.G. Reznitskikh, Phys. Low-Dimens. Str. 1-2, 89 (1997).

6. E.I. Rashba, Phys. Rev. B62, R16267 (2000).

7. R.M. Potok, J.A. Folk, C.M. Markus, and V. Umanski, Phys. Rev. Lett. 89, 266602 (2002).

8. S.H. Chun, S.J. Potashnik, K.C. Ku, P. Schiffer, and N. Samarth, Phys. Rev. B66,100408 (2002).

9. A.T. Hanbicki, B.T. Jonker, G. Itkos, G. Kioseoglou, and A. Petrou, Appl. Phys. Lett. 80, 1240 (2002).

10. A. Kadigrobov, Z. Ivanov, T. Claeson, R.I. Shekhter, and M. Jonson, Europhys. Lett. 67, 948 (2004).

11. Yu.V. Gulyaev, P.E. Zilberman, A.I. Krikunov, A.I. Panas, and E.M. Epstein, JETP Lett. 85, 160 (2007).

12. X.R. Wang, Research Letters in Physics 2008, Article ID434936 (2008).

13. A.M. Kadigrobov, R.I. Shekhter, M. Jonson, S.I. Kulinich, O.P. Balkashin, V.V. Fisun, Yu.G. Naidyuk, I.K. Yanson, S. Andersson, and V. Korenivski, New J. Phys. 13, 023007 (2011).

14. Yu.V. Gulyaev, P.E. Zilberman, I.V. Malikov, A.I. Panas, S.G. Chigarev, and E.M. Epstein, Pis'ma v ZhETF 93, 289 (2011).

15. S.V. Vonsovskii, Magnetism, Vol. 2, § 3, p. 635, Wiley, New York (1974); H.J. Zeiger and Pratt, Magnetic Interactions in Solids, Clarendon Press, Oxford (1973).

16. I.O. Kulik, A.N. Omel'yanchuk, and R.I. Shekhter, Fiz. Nizk. Temp. 3, 1543 (1977) [Sov. J. Low Temp. Phys. 3, 740 (1977)]. 
17. I.O. Kulik, R.I. Shekhter, and A.G. Shkorbatov, Sov. Phys. JETP 54, 1130 (1981).

18. O.P. Balkashin, V.V. Fisun, I.K. Yanson, L.Yu. Triputen', A. Konovalenko, and V. Korenivski, Phys. Rev. B79, 092419 (2009); O.P. Balkashin, V.V. Fisun, I.K. Yanson, L.Yu. Triputen', A. Konovalenko, and V. Korenivski, Fiz.
Nizk. Temp. 35, 879 (2009) [Low Temp. Phys. 35, 693 (2009)].

19. R.I. Shekhter, A.M. Kadigrobov, M. Jonson, E.I. Smotrova, A.I. Nosich, and V. Korenivski, arXiv:1102.2721 (unpublished). 